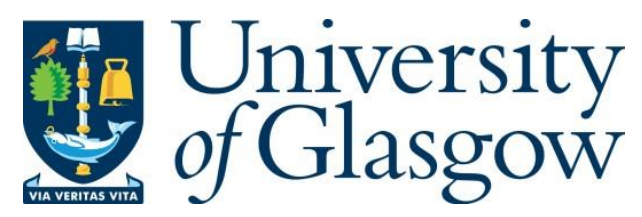

Rolinski, O. J., McLaughlin, D., Birch, D. J.S., and Vyshemirsky, V. (2016) Resolving environmental microheterogeneity and dielectric relaxation in fluorescence kinetics of protein. Methods and Applications in Fluorescence, 4(2), 024001.

There may be differences between this version and the published version. You are advised to consult the publisher's version if you wish to cite from it.

http://eprints.gla.ac.uk/135826/

Deposited on: 7 March 2017

Enlighten - Research publications by members of the University of Glasgow http://eprints.gla.ac.uk 


\title{
Resolving environmental microheterogeneity and dielectric relaxation in fluorescence kinetics of protein
}

\author{
Olaf J. Rolinski ${ }^{1}$, Damien McLaughlin ${ }^{1}$, David J.S. Birch ${ }^{1}$ and Vladislav Vyshemirsky ${ }^{2}$ \\ ${ }^{1}$ Photophysics Group, Centre for Molecular Nanometrology, Department of Physics, Scottish Universities Physics \\ Alliance, University of Strathclyde, 107 Rottenrow, Glasgow G4 0NG, UK \\ ${ }^{2}$ School of Mathematics and Statistics, University of Glasgow, Glasgow G12 8QQ, UK
}

Keywords: protein fluorescence, microheterogeneity, dielectric relaxation, global analysis, human serum albu$\min$.

\begin{abstract}
The fluorescence intensity decay of protein is easily measurable and reports on the intrinsic fluorophore-local environment interactions on the sub-nm spatial and sub-ns temporal scales, which are consistent with protein activity in numerous biomedical and industrial processes. This makes time-resolved fluorescence a perfect tool for understanding, monitoring and controlling these processes at the molecular level, but the complexity of the decay, which has been traditionally fitted to multi-exponential functions, has hampered the development of this technique over the last few decades. Using the example of tryptophan in HSA we present the alternative to the conventional approach to modelling intrinsic florescence intensity decay in protein where the key factors determining fluorescence decay, i.e. the excited-state depopulation and the dielectric relaxation [Toptygin, Brand, Chem.Phys.Lett. (2000) 322:496-502], are represented by the individual relaxation functions. This allows quantification of the both effects separately by determining their parameters from the global analysis of a series of fluorescence intensity decays measured at different detection wavelengths. Moreover, certain pairs of the recovered parameters of tryptophan were found to be correlated, indicating the influence of the dielectric relaxation on the transient rate of the electronic transitions. In this context the potential for the dual excited state depopulation /dielectric relaxation fluorescence lifetime sensing is discussed.
\end{abstract}




\section{Introduction}

The fluorescence intensity decay of the intrinsic fluorophore in protein is complex due to the microheterogeneity of environment, the fluorophore-environment dielectric relaxation and the specific interactions of the fluorophore. Therefore protein fluorescence decays are non-exponential and are traditionally parameterized by the linear combinations of exponentials. Here we reject this approximation and introduce new general relaxation functions for modelling fluorescence decays, which implies a new approach to data analysis and allows clear interpretation of the observed complexity as a compilation of two simple relaxation processes.

As demonstrated in numerous experiments, protein fluorescence decay fits well to multi-exponential functions and shows the lifetime-wavelength correlation, that is, the mean decay lifetime increases with the detection wavelengths. Two contradicting views explaining these observations are explored: the model of rotamers and the model of dielectric relaxation. The rotamer view [1-5] assumes the existence of discrete ground-state subpopulations of fluorophores (rotamers), each exhibiting a distinct decay time. In the dielectric relaxation model [6,7], the observed multi-exponentiality is explained by fluorescence spectrum shifts to longer wavelengths on a nanosecond time scale. Both models have contributed to a better understanding of protein photophysics, but they are not free from limitations. For example the rotamer model does not explain the wavelength-lifetime correlation (although recent quantum-molecular mechanics simulations of some peptides [8] have suggested that the existence of multiple rotamers can lead to the lifetime-wavelength correlation), and the dielectric relaxation model does not explain the lack of the common appearance of negative amplitudes in protein decay, which is predicted by this model.

More recently descriptions combining the conformational heterogeneity (rotamers) with dielectric relaxation have emerged [9-16]. In the original paper by Toptygin and Brand [9], the spectrally- and time-resolved fluorescence emission $I_{v}(v, t)[9-12]$, is written as

$$
I_{v}(v, t)=v^{3} S\left(v-v_{c}(t)\right) D(t)
$$

where $S(v)$ is the vibration envelope representing the fluorescence transitions, $D(t)$ is the decay of the excitedstate population and $v_{c}(t)$ is the time variation of the spectral centroid. Traditionally, the functions $D(t)$ and $v_{c}(t)$ are parameterised by linear combinations of exponentials and $S(v)$ is expanded in a Taylor series, which leads to a multi-exponential function 


$$
I_{v}(v, t)=\sum_{i=1}^{N} a_{i}(v) \exp \left(-t / \tau_{i}(v)\right)
$$

Different experimental approaches exploring the eqtn (2) [9-16] have been developed to reveal spectral and timeresolved fluorescence, including recent studies of tryptophan (Trp) kinetics in monellin [16], where Trp has been replaced by 5 -fluorotryptophan, which shows reduced effects of the heterogeneity of the environment and thus provides a clearer view of the dielectric relaxation. Another technique that uses the multi-exponential representation of Trp fluorescence decays for determining protein solvation dynamics has been introduced by Zhong and co-workers $[17,18]$. In this approach the decay is assumed to be a sum of two multi-exponential functions. The components of the first function (representing the dielectric relaxation) are identified by the positive preexponential factors for the decays measured at the blue side of the emission spectrum, which then become negative at the red side of the spectrum. The pre-exponential coefficients of the second function (representing the decay of the excited population) are always positive. This separation of the dielectric relaxation and the excited-

state depopulation is then used to construct the normalised solvation correlation function, which is not affected by the potential heterogeneity of the environment and resulting non-exponential population decay.

In our opinion, using the multi-exponential approximation of the fluorescence decay may lead to the loss or missinterpretation of information within raw fluorescence decay. This is because multi-exponentiality may not be supported by physical model. On the other hand, the model supporting multi-exponentiality, i.e. the existence of few subsystems of distinct lifetimes, is unlikely to be adequate in the complex biological environments that are further complicated by dielectric relaxation. In this case the continuous distribution of lifetimes seems to give a more realistic picture.

In this paper we attempt a new approach to resolve the excited-state heterogeneity and dielectric relaxation. We propose and explore the alternative model of $I_{v}(v, t)$ based on the new, general representations of the $D(t)$ and $v_{c}(t)$ relaxation functions derived from the principles of the non-equilibrium statistical physics.

_We have already demonstrated for human serum albumin [19], that its Trp fluorescence decay can be represented by the general relaxation function

$$
I_{\alpha, \kappa}(t)=\exp \left[-\frac{1}{\kappa} \int_{0}^{\kappa\left(t / \tau_{0}\right)^{\alpha}}(1-\exp [-1 / x]) d x\right]
$$


where $\tau_{0}$ is the fluorescence lifetime of the amino acid while the parameters $\alpha$ and $\kappa$ determine the stable distributions of fluorescence transition rates. The relaxation function (3) has been originally derived in the theory describing dipole kinetics in solid materials [20] and provides a more general than exponential description of fluorescence kinetics, which can be particularly useful for interpreting fluorescence response in complex environments. The dependence (3) is a two-power-law relaxation response, and it is worth noting that the commonly used exponentials $(\alpha=1, \kappa=0)$, stretched-exponentials $\sim \exp \left[-(\mathrm{t} / \tau)^{\alpha}\right][21](\alpha<1, \kappa=0)$ and Becquerel functions $(1+\kappa(\mathrm{t} / \tau))^{-1 / \kappa}$ [22] $(\alpha=1, \kappa>0)$, are but special cases of the eqtn (3).

We have also used the model (3) to represent the fluorescence decay of tyrosine in beta-amyloid peptides during their aggregation (known to lead to Alzheimer's disease) [23]. The analysis of the changes in parameters of model (3), and specifically in the parameter $\alpha$ becoming larger than 1, which is not possible in the excited-state kinetics, has led us to the conclusion that the experimental decay may be influenced by the excited population being interfered with by the dielectric relaxation; this being more frequent than would be anticipated from the lack of appearance of negative components in multi-exponential analysis.

Here we research the hypothesis that the individual fluorescence decay of intrinsic fluorophore in protein, measured at different wavelengths and temperatures, do not only follow the general model (3), which has been already partially demonstrated [19], but are also consistent with a more detailed model decay (1) with the $D(t)$ and $v_{c}(t)$ relaxation functions given by the general forms of type (3). We apply the global analysis to a series of fluorescence decays of Trp in HSA measured at different detection wavelengths, and extract the parameters of the both relaxation processes. It is assumed that $I_{v}(v, t)$ is given by

$$
I_{v}(v, t)=v^{3} S\left(v-v_{\infty}-\left(v_{0}-v_{\infty}\right) \Phi_{s}(t)\right) \Phi_{f}(t)
$$

where $\Phi_{f}(t)$ and $\Phi_{s}(t)$ are the new fluorescence and solvation relaxation functions:

$$
\Phi_{f}(t)=\exp \left[-\frac{1}{\kappa_{f}} \int_{0}^{\kappa_{f}\left(t / \tau_{f}\right)^{\alpha_{f}}}(1-\exp [-1 / x]) d x\right]
$$

and

$$
\Phi_{s}(t)=\exp \left[-\left(\frac{t}{\tau_{s}}\right)^{\alpha_{s}}\right]
$$


where $\Phi_{f}(0)=\Phi_{s}(0)=1$ and $\Phi_{f}(\infty)=\Phi_{s}(\infty)=0$. The parameters $\tau_{f}, \alpha_{f}$ and $\kappa_{f}$ characterise the decay of the excited state population and enable high flexibility in representing the transient fluorescence decay rates $k_{f}(t)=-d\left(\ln \Phi_{f}(t)\right) / d t$, including a non-monotonic behaviour [23], while $\tau_{s}$ and $\alpha_{s}$ describe the dielectric relaxation. In this approach both sets of the parameters are expected to be independent of the fluorescence decay detection wavelengths/ wavenumbers, because the dependence of $I_{v}(v, t)$ on $v$ is only that specified explicitly in the formula (4). However, we do not exclude the existence of potential correlations between the $f$ - and $s$-type parameters, as the dielectric relaxation may influence the population decay. Indeed, the Trp transient decay rate $k_{f}(t)$ may change when $\operatorname{Trp}$ reorientates in respect to its surroundings. Therefore the potential correlations between the recovered parameters are also investigated. The function $S(v(t))$ representing the distribution of fluorescence transitions has been modelled by the Gaussian profile. HSA has been selected as a model protein that has one Trp and shows only Trp fluorescence when excited at $295 \mathrm{~nm}$. Nethertheless, HSA fluorescence decay is known to be complex due to heterogeneity of Trp environments and because the typical lyophilized HSA sample contains up to $10 \%$ higher order aggregates formed via disulfide linkage $[24,25]$. The variety of possible Trp environments leads to the nonexponential decay of the excited population. This combines with the dielectric relaxation and results in a complex decay. In our approach we aim to resolve these two effects.

\section{Experimental}

HSA and the phosphate buffer were purchased from Sigma-Aldrich (Poole, UK). The $3 \times 10^{-5} \mathrm{M}$ HSA solution in 0.01 M phosphate buffer, pH7.4 has been prepared on the day of measurements. The time-correlated single photon counting (TCSPC)-based the Fluorocube fluorescence lifetime system (Horiba Jobin Yvon IBH, Glasgow, UK) has been used to record the fluorescence decays. An AlGaN version of a pulse light emitting diode working at $295 \mathrm{~nm}$ [26] and $1 \mathrm{MHz}$ repetition rate has been used to excite the Trp directly. A polarizer of vertical orientation in the excitation channel and the other at the magic angle in the detection channel were used. Measurements were taken for the temperatures $10^{\circ} \mathrm{C}$ to $60^{\circ} \mathrm{C}$ with the 10 degrees steps by using the temperature-controlled sample holder connected to a Neslab RTE11 thermostat (Thermo Scientific UK). For each temperature, the detection wavelengths have been changed from $330 \mathrm{~nm}$ to $360 \mathrm{~nm}$ with $5 \mathrm{~nm}$ increments. 
Fluorescence decays were analysed by two deconvolution procedures, first assuming the model given by eqtn (3), the second explored the dependence (4). In both cases, the experimental decays were expressed as

$$
F(t)=a+b L(t+\Delta)+c \int_{0}^{t} L\left(t+\Delta-t^{\prime}\right) I\left(t^{\prime}\right) d t^{\prime}
$$

where $L(t)$ is the prompt excitation function, $a, b$, and $c$ are the background level, contribution of the scattered light and the scaling parameter, respectively, and $\Delta$ is the time-shift between the prompt and decay curves due to the colour effect of the light detector and finite channel width of the time axis. $I(t)$ represents the model decay given by eqtn (3) or (4).

The parameters of model (3) were obtained from the deconvolution procedure described elsewhere [19] by minimising the $\chi^{2}$ goodness-of-fit criterion. The model (4) has been investigated by global deconvolution analysis, where a number of the decays measured for the different detection wavelengths were fitted jointly.

Global analysis was performed by optimisation of the $\chi^{2}$ goodness of fit criterion over the full set of data from all available fluorescence profiles measured at a range of wavelengths at the same time. The function $S(v(t))$ representing the distribution of fluorescence transitions has been modelled by the Gaussian distribution. The parameters of model (4) remained common for all detection wavelengths. The parameters of the functions (5) and (6) were fitted during the procedure, while the peak initial and final wavenumbers $v_{0}=28635 \mathrm{~cm}^{-1}$ and $v_{\infty}=28247 \mathrm{~cm}^{-1}$ were estimated from the Otosu et al. paper [15] for the HSA data studied at $\mathrm{pH} 7$ in order to ensure the consistence of our analysis with the former precise measurements of the transient emission spectra of HSA. The technical parameters of (7) ( $a, b, c$ and $\Delta$ ) were fitted individually for each decay. The $\chi^{2}$ goodness-of-fit criterion was minimised using the Nelder-Mead optimisation method [27]. Due to the possible existence of local minima, the optimisation procedure was performed at least 12 times using randomly generated starting values of the model parameters, and the best result was selected as the final estimate of model parameters. Overall computations to obtain such an estimate with 12 repetitions take on average 4 hours using a typical desktop computer capable of 96 GFLOPS peak performance. 


\section{Results and discussion}

The fluorescence decays of Trp in HSA were recorded at different detection wavelengths across the Trp fluorescence spectrum and at different temperatures. The $\chi^{2}$ values obtained while fitting the model (3) to the data in no case exceeded 1.05, indicating a very good fit for all the decays. Although fitting a 3 -exponential model to HSA data is known to give similar values of $\chi^{2}$, the model (3) is still more adequate for the measured decay by means of the Akaike Information Criterion (AIC) [28], which measures the appropriateness of the model decay on the basis of the both unreduced $\chi^{2}$ value and the number of parameters $k$ of the model function $A I C=\chi^{2}+2 k$. The smaller value of AIC indicates the more adequate model. In our case the AIC for model (3) was smaller than that for the 3-exponential function for the most ( 75\%) decays. The average value of AIC for (3) was 2725.3 , while for a 3-exponential decay it was 2767.2.

The recovered parameters $\tau, \alpha$ and $\kappa$ (Fig. 1) respond to changes in the temperature $\mathrm{T}$ and the detection wavelength $\lambda$, reflecting the photophysics of Trp in HSA. An increase in $\tau$ with $\lambda$ confirms the wavelength-lifetime correlation, which is consistent with the presence of dielectric relaxation, while the drop of $\tau$ with the temperature reflects more intensive collisional quenching of Trp by the surroundings. Changes in $\alpha$ and $\kappa$ (Fig.1.b and c.) are similar to those observed for $\tau$ and provide additional information. For example, the $\alpha$ values lower than 1 indicate a non-Debye (non-exponential) decay, which results from a broader (heavy-tailed) [19,20] distribution of the transition rates. Here we notice that the dominant stretched-exponential nature of the decay $(\alpha<1, \kappa \rightarrow 0)$, observed at the shorter detection wavelengths and lower temperatures, gradually becomes closer to the Becquereltype $(\alpha \rightarrow 1, \kappa>0)$ at longer wavelengths and higher temperatures. Such convolved character of the recovered decays parameters is consistent with more than one relaxation process contributing to the experimental decays.

To test our hypothesis we have fitted the double-relaxation model $I_{v}(v, t)$ (eqtn $\left.(4)\right)$ and applied global analysis for the sets of decays measured at different wavelengths and recovered the parameters of the functions (5) and (6).

Figure 2 shows the example of the global fitting of a series of the decays of HSA sample at $50^{\circ} \mathrm{C}$ measured at different wavelengths. 
The results for all temperatures are summarised in Fig.3. The $\chi^{2}$ values (shown as numbers at the experimental points in Fig. 3a) are acceptable over the whole range of temperatures. Although they do not show a very good fit at lower temperatures, which is discussed later, the results of the global analysis confirm the general validity of the model given by eqtn (4).

The parameters $\tau_{F}$ and $\alpha_{F}$, responsible for the decay of the excited-state population, decrease slowly with temperature. This is expected as the result of increased collisional quenching and broader range of molecular orientations (thus transition rates), respectively, however the $\kappa_{F}$ parameter has been found to be negligibly small. This finding leads to the conclusion, that the relaxation of the excited-states of Trp in HSA can be described by a single stretched-exponential function and the environment heterogeneity of the Trp can be characterised by only one parameter, the index of stability $\alpha_{F}$, which determines the stable distribution of the transition rates $f_{\alpha F}(k)$ of Trp.

The significant changes in the parameters $\tau_{S}$ and $\alpha_{S}$ of the dielectric relaxation function $\Phi_{S}(t)$, as compared to the slower drop in the $\Phi_{F}(t)$ parameters suggests, that between $30^{\circ} \mathrm{C}$ and $40^{\circ} \mathrm{C}$ there is a change in HSA conformation, which substantially affects the rate of dielectric relaxation, but only slightly (through the normal temperature effect) the rate of fluorescence decay. This suggests the change of the whole HSA configuration, but not a change in Trp's local environment. As $\tau_{S}$ decreases, this conformational change must lead to faster relaxation.

Figure 4 presents the resulting $\Phi_{F}(t)$ and $\Phi_{S}(t)$ relaxation functions for different temperatures. The $\Phi_{F}(t)$ dependence shows relatively weak accelerating effect of temperature on the decay of the excited-state population, while the $\Phi_{S}(t)$ demonstrates a rapid increase in the rate of dielectric relaxation at higher temperatures. This is consistent with the phase transition of HSA molecules occurring between 30 and $40^{\circ} \mathrm{C}$. After this transition, the dielectric relaxation is much faster and its rate still increases with temperature. Again, it is worth noting, that this process, which involves a whole HSA molecule, is not directly reflected in the fluorescence decay rates of Tyr, confirming our suggestion that there is no substantial change in the Tyr immediate surroundings. This is consistent with the HSA studies by Otosu et al. [15], who, by using the time-resolved area-normalised emission spectra (TRANES) method, noticed that the local conformational transition near Trp in HSA and the entire conformational change of the whole protein have different characteristics.

Figures 4.a. and b. also show that at lower temperatures dielectric relaxation accompanies the whole process of fluorescence decay, while at higher temperatures the dielectric relaxation is completed at the early stages of the 
fluorescence decay. This may explain the observed poorer values of the $\chi^{2}$ parameters at lower temperatures. Indeed, in the model (4) the influence of the dielectric relaxation on the experimental fluorescence decays is represented only as the result of the time-dependent Stokes shift of the emission spectrum. In the real system, the role of dielectric relaxation is much more specific, because the probabilities for radiative and non-radiative transitions of the excited molecule depend on the local environment, which changes continuously during dielectric relaxation. This effect can be especially significant in heterogeneous environments for tryptophan as would be experienced with protein. Therefore dielectric relaxation is likely to affect the decay of the population of excited fluorophores, which can be manifested in the correlations between the parameters characterising both relaxation processes. Our data confirm the existence of these correlations. Table 1 shows that $\tau_{S}$ is correlated with both $\tau_{F}$ and $\alpha_{F}$ at $5 \%$ significance level and corresponding coefficients indicate relatively strong positive correlation. Additionally, there is very strong correlation between $\tau_{F}$ and $\alpha_{F}$ on their own, while there is no evidence of correlation in other pairs of parameters. This correlation analysis only considers linear relationships and is based on a small sample of $n=6$ parameter values estimated for the experiments performed at different temperatures.

\section{Conclusion}

The proposed spectrally- and time-resolved fluorescence model (eqtns (4-6)) enables separation of the two processes dominating the intrinsic fluorescence decay of protein: heterogeneity of the excited-state decay and dielectric relaxation. Combining this model with global analysis allows determination of the parameters of dualrelaxation, which paves the way to the dual relaxation-based sensing of heterogeneous environments with broad potential applications wherever protein fluorescence occurs. Moreover, studying correlations between the recovered parameters of both relaxations may offer new information on protein activity.

In this specific research we have found, that the conformational change, which the HSA undergoes between 30 and $40^{\circ} \mathrm{C}$, accelerates its dielectric relaxation, but does not affect the decay of Trp excited-state population, thus it does not occur in the Trp local environment. Revealing the parameters of both relaxations by applying this approach to other proteins, e.g. those associated with the onset of certain diseases, could offer a new range of information on the structural processes involved on the nm scale. 


\section{References:}

1. Donzel B, Gaudcho P, Wahl P 1974 Study of conformation in excited state of 2 tryptohanyl diketopiperazines J.Am.Chem.Soc. 96(3) 801-808.

2. Szabo AG, Rayner DM 1980 Fluorescence decay of tryptophan conformers in aqueous solution J.Am.Chem.Soc. 102(2) 554-563.

3. Adams PD, Chen Y, Ma K, Zagorski MG, Sönnichsen FD, et al. 2002 Intramolecular quenching of tryptophan fluorescence by the peptide bond in cyclic hexapeptides JACS 124(31) 9278-9286.

4. Julien O, Wang G, Jonckheer A, Engelborghs Y, Stykes BD 2012 Tryptophan side chain conformers monitored by NMR and time-resolved fluorescence spectroscopies Proteins 80 239-245.

5. Gasymov OK, Abduragimov AR, Glasgow BJ 2012 Tryptophan rotamer distribution revealed for the $\alpha$-helix in tear lipocalin by site-directed tryptophan fluorescence J.Phys.Chem.B 116(45) 13381-13388.

6. J.R.Lakowicz 2000 On spectral relaxation in proteins. Photochem.Photobiol. 72(4) 421-437.

\section{Lakowicz JR Principles of fluorescence spectroscopy (Springer, New York, 2006)}

8. Pan C-P, Muino PL, Barkley MD, Callis PR 2011 Correlation of tryptophan fluorescence spectral shifts and lifetimes arising directly from heterogeneous environment J.Phys.Chem.B 115 3245-3253.

9. Toptygin D, Brand L 2000 Spectrally- and time-resolved fluorescence emission of indole during solvent relaxation: a quantitative model Chem.Phys.Lett. 322 496-502.

10. Toptygin D, Savtchenko R.S, Meadow ND, Brand L 2001 Homogeneous spectrally- and time-resolved fluorescence emission from single tryptophan mutants of IIA ${ }^{\text {Glc }}$ protein J.Phys.Chem.B. 105 2043-2055.

11. Toptygin D, Gronenborn AM, Brand L 2006 Nanosecond relaxation dynamics of protein GB1 identified by the time-dependent red shift in the fluorescence of tryptophan and 5-fluorotryptophan J.Phys.Chem.B $11026292-$ 26302.

12. Toptygin D 2014 Analysis of time-dependent red shifts in fluorescence emission from tryptophan residues in proteins. Fluorescence spectroscopy and microscopy: Methods and protocols, Methods in Molecular Biology vol.1075, edts Engelborghs Y, Visser AJWG (Springer Science +Bussiness Media), pp 215-256.

13. Otosu T, Nishimoto E, Yamashita S 2007 Spectrally and time-resolved fluorescence spectroscopic study on melittin-calmodulin interaction. J.Biochem. 142 655-661.

14. Otosu T, Nishimoto E, Yamashita S 2009 Fluorescence decay characteristics of indole compounds revealed by time-resolved area-normalized emission spectroscopy J.Phys.Chem.A. 113 2847-2853. 
15. Otosu T, Nishimoto E, Yamashita S 2010 Multiple conformational state of human serum albumin around single tryptophan residue at various pH revealed by time-resolved fluorescence spectroscopy J.Biochem. 147(2) 191200.

16. Xu J, Chen B, Callis P, Muino PL, Rozeboom H, Broos J, Toptygin D, Brand L, Knutson JR 2015 Picosecond fluorescence dynamics of tryptophan and 5-fluorotryptophan in monellin: Slow water-protein relaxation unmasked. J.Phys.Chem. B 119 4230-4239.

17. Lu W, Kim J, Oiu W, Zhong D 2004 Femtosecond studies of tryptophan solvation: correlation function and water dynamics at lipid surfaces. Chem.Phys.Letts 388 120-126.

18. Qin Y, Chang C-W, Wang L, Zhong D 2012 Validation of response function construction and probing heterogeneous protein hydration by intrinsic tryptophan J.Phys.Chem. B 116 13320-13330.

19. Rolinski OJ, Vyshemirsky V 2014 Fluorescence kinetics of tryptophan in a heterogeneous environment Methods Appl.Fluoresc. 2045002 (13pp).

20. Weron K, Jurlewicz K 1993 2-forms of self-similarity as a fundamental feature of the power-law dielectric response. J.Phys.A: Math.Gen. 26 395-410.

21. Berberan-Santos MN, Valeur B (2005) Mathematical functions for the analysis of luminescence decays with underlying distributions. 1.Kohlraush decay function (stretched exponential). Chem.Phys. 315:171-182.

22. Berberan-Santos MN, Valeur B 2005 Mathematical functions for the analysis of luminescence decays with underlying distributions. 2.Becquerel (compressed hyperbola) and related decay functions Chem.Phys. 317 57-62.

23. Rolinski OJ, Wellbrock T, Birch DJSB, Vyshemirsky V 2015 Tyrosine photophysics during the early stages of $\beta$-amyloid aggregation leading to Alzheimer's J.Phys.Chem.Lett. 6 3116-3120.

24. Constantino HR, Shieh L, Klibanov AM, Langer L 1997 Heterogeneity of serum albumin samples with respect to solid-state aggregation via thiol-disulfide interchange: implications for sustained release from polymers J.Control.Release 44 255-261.

25. James NG, Ross JA, Stefl M, Jameson DM 2010 Applications of phasor plots to in vitro protein studies Anal.Biochem. 410 70-76.

26. McGuinness CD, Sagoo K, McLoskey D, Birch DJSB 2005 Selective excitation of tryptophan fluorescence decay in proteins using a subnanosecond $295 \mathrm{~nm}$ light-emitting diode and time-correlated single-photon counting Appl. Phys. Lett. 86261911.

27. Nelder AJ, Mead R 1965 A simplex method for function minimization. Computer Journal 7 308-313. 
28. Burnham KP, Anderson DR Model selection and multimodel interference: A practical information-theoretic approach, $2^{\text {nd }}$ ed. (Springer: New York,2002).

Table 1. Correlation between parameters (those significant at 5\% level are in bold)

\begin{tabular}{|c|c|c|c|c|c|}
\hline & $\tau_{\mathrm{F}}$ & $\alpha_{\mathrm{F}}$ & $\kappa_{\mathrm{F}}$ & $\tau_{\mathrm{S}}$ & $\alpha_{S}$ \\
\hline$\tau_{\mathrm{F}}$ & 1 & 0.9873 & 0.7171 & 0.8913 & -0.6284 \\
\hline$\alpha_{F}$ & & 1 & 0.6671 & 0.8543 & -0.6976 \\
\hline$\kappa_{\mathrm{F}}$ & & & 1 & 0.4873 & 0.0318 \\
\hline$\tau_{\mathrm{S}}$ & & & & 1 & -0.5960 \\
\hline$\alpha_{S}$ & & & & & 1 \\
\hline \multicolumn{6}{|c|}{ p-values (significance of the correlation test) } \\
\hline$\tau_{\mathrm{F}}$ & & 0.0003 & 0.1088 & 0.0171 & 0.1815 \\
\hline$\alpha_{\mathrm{F}}$ & & & 0.1478 & $\mathbf{0 . 0 3 0 3}$ & 0.1234 \\
\hline$\kappa_{\mathrm{F}}$ & & & & 0.3269 & 0.9524 \\
\hline$\tau_{\mathrm{S}}$ & & & & & 0.2119 \\
\hline$\alpha_{S}$ & & & & & \\
\hline
\end{tabular}


Figures:

a.



b.

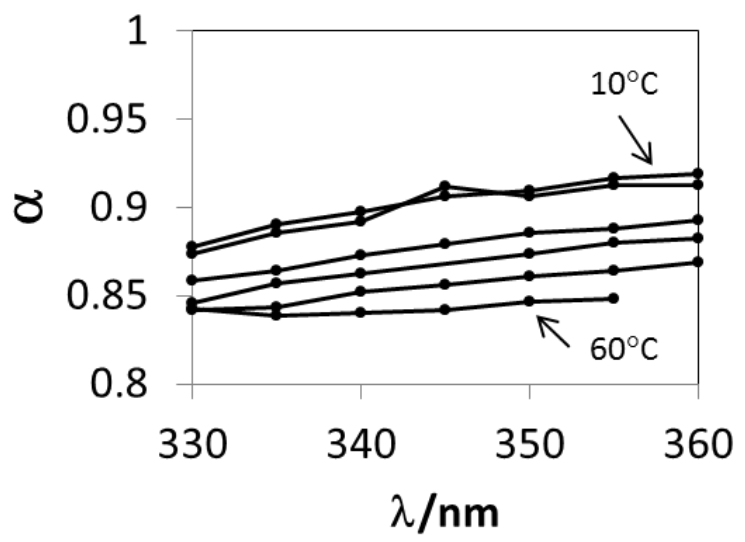

C.

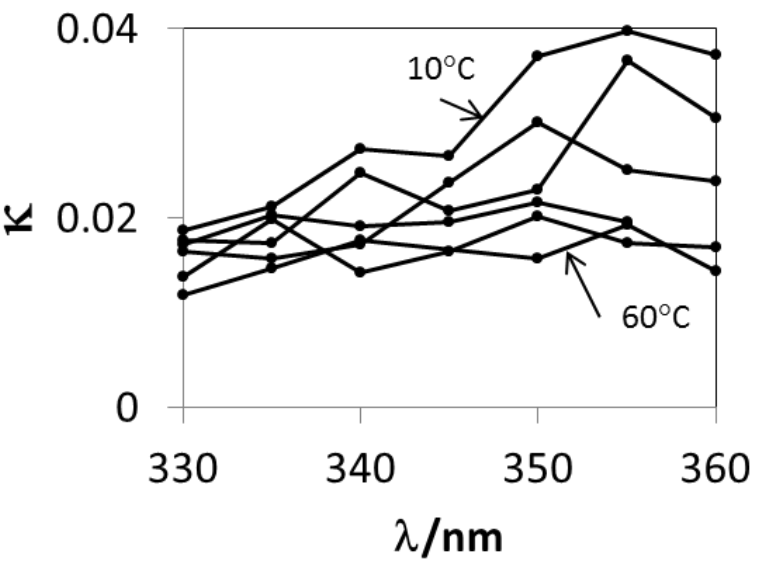

Fig.1. The $\tau$ (a.), $\alpha$ (b.) and $\kappa$ (c.) parameters of the model decay (3) recovered from the HSA fluorescence decays measured at different wavelengths at temperatures between $10^{\circ} \mathrm{C}$ and $60^{\circ} \mathrm{C}$. All $\chi^{2}$ values in the range 0.95 1.05 . 


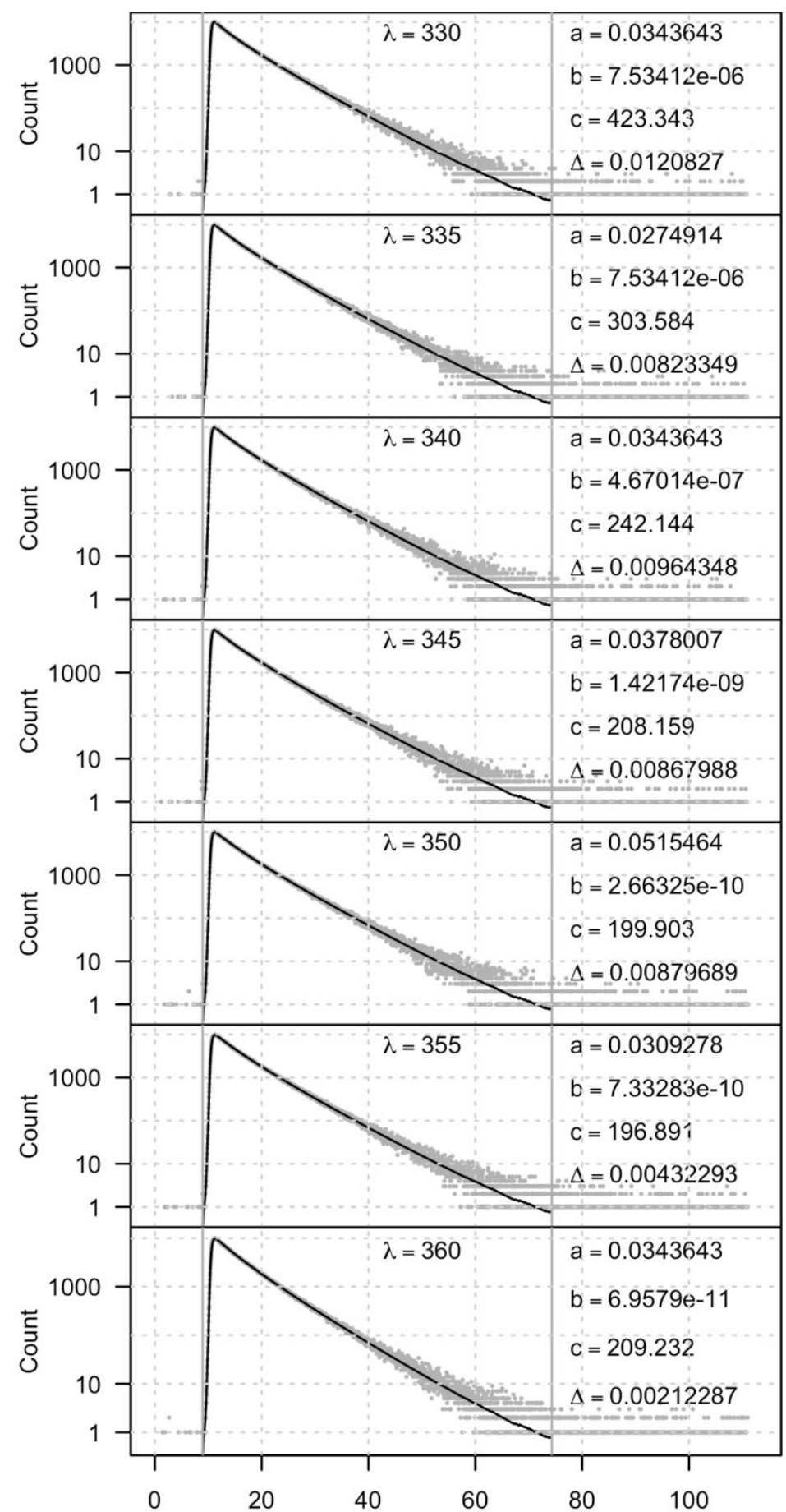

Time/ns

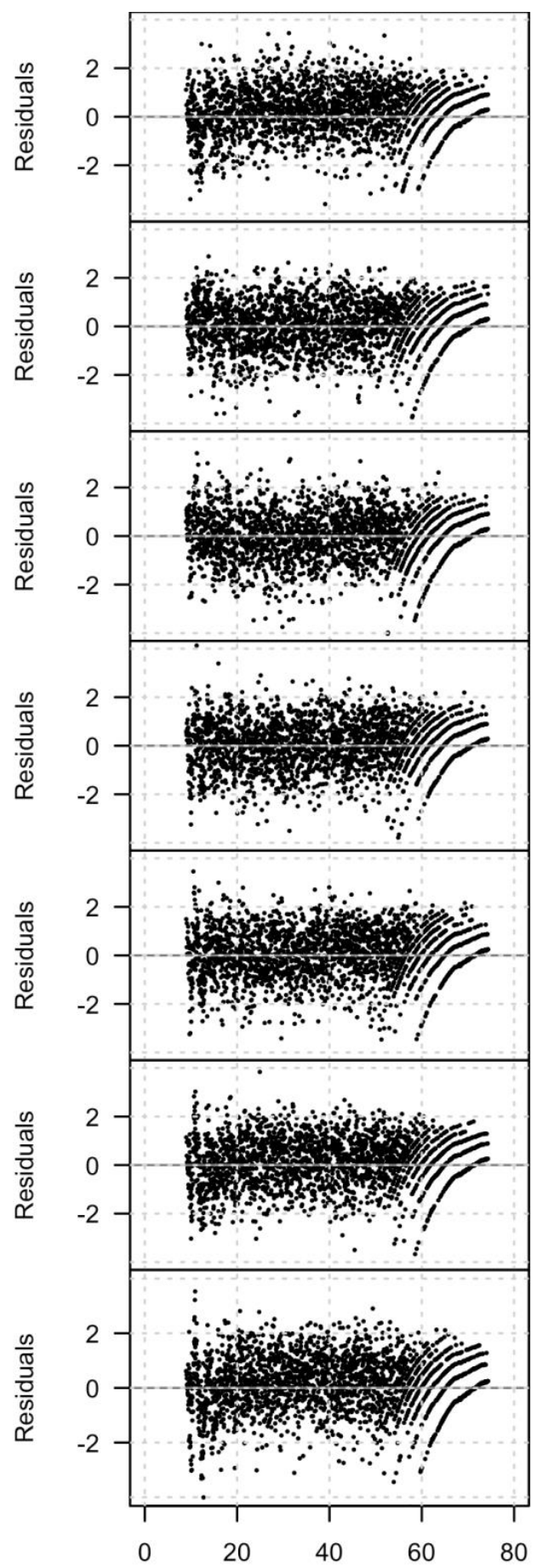

Time/ns

$$
\begin{aligned}
& v_{0}=28635 \\
& v_{\infty}=28247 \\
& \sigma=1600 \\
& \chi^{2}=1.07995
\end{aligned}
$$

Fig.2. The raw decays of HSA fluorescence at $50^{\circ} \mathrm{C}$ measured at different wavelengths, the fitted functions and the distribution of residuals, together with the parameters of the model defined by eqtn (4) recovered in the global analysis. Note that the model parameters are common for the all set of decays, and only technical parameters a, b, $\mathrm{c}$ and $\Delta$ are specific to each decay. 

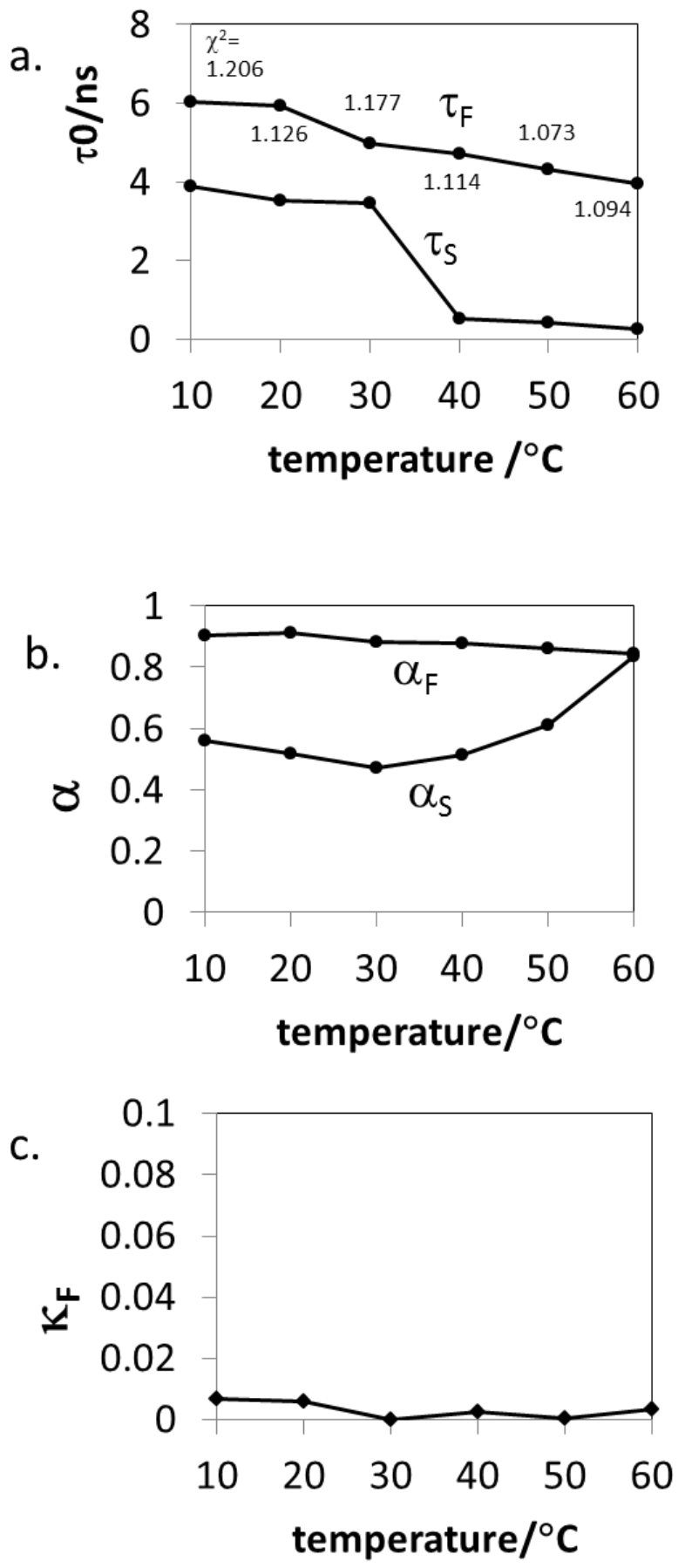

Fig.3. Temperature dependence of the parameters $\tau_{\mathrm{F}}$ and $\tau_{\mathrm{S}}(\mathrm{a}),. \alpha_{\mathrm{F}}$ and $\alpha_{\mathrm{S}}$ (b.) and $\kappa_{\mathrm{F}}$ (c.) obtained for the double-relaxation model (4) of fluorescence kinetics, from the global analysis of the series of decays measured at the different detection wavelengths. 



Fig.4. The normalised decays of the excited-state populations $\Phi_{\mathrm{f}}(\mathrm{t})(\mathrm{a}$.$) , and the dielectric relaxation functions$ $\Phi_{\mathrm{s}}(\mathrm{t})(\mathrm{b}$.$) at different temperatures.$ 